\title{
Advances in neuromyelitis optica
}

Avanços em neuromielite óptica

\section{Emerson L. Gasparetto ${ }^{1}$, Fernanda C. Rueda Lopes ${ }^{2}$}

Neuromyelitis optica (NMO) is considered a demyelinating disorder, predominantly affecting the optic nerveand thespinal cord. However the diseaseconcept has undergonechanges over the past decade. In theneurological field, NMO is nolonger considered as being a multiple sclerosis (MS) subtype, being currently diagnosed as a disease itself, with a differentiated clinical course, besides different prognosis and treatment as compared with MS. In what regards the immunological aspect, the discovery of the central nervous system water channels (aquaporin) involvement by an autoantibody called anti-AQP4 was a key factor leading to the differentiation of the disease. The attack by autoimmune complexes to areas containing water channels, particularly thoseadjacent to ventricular regions, in the optic nerves and around the central channel of the spinal cord, causes necrosis in such regions, leading tomore severelesions which are more difficult to heal than MS lesions.

All such discoveries were essential for the evolvement of radiological evaluation in NMO. The involvement of the brain in NMO has been increasingly investigated. Previously, the criteria for diagnosis of NMO established that cranial magnetic resonance imaging (MRI) should benormal. Currently, such criteria indude the presence of lesions at cranial MRI which donot meet the imaging criteria for MS. At MRI, intracranial lesions are poorly specific, but are frequently found, affecting up to $90 \%$ of the cases. However, about 8-10\% of the patients present moresevere brain lesions, with typical images from affected regions which are rich in APQ4 channels, such as those around the third ventricle, the periaqueductal gray matter, hypothalamus and periventricular region ${ }^{(\mathbf{1})}$.

\footnotetext{
1. Associate Professor of Radiology (Neuroimaging), Universidade Federal do Rio de J aneiro (UFRJ ), Neuroradiologist at Clínica de Diagnóstico Por Imagem (CDPI)/DASA, Rio de J aneiro, RJ , Brazil. E-mail: egasparetto@gmail.com

2. Fellow PhD degree, Program of Post-graduation in Medicine (Radiology), Universidade Federal do Rio de J aneiro (UFRJ ), Neuroradiologist at Clínica de Diagnóstico Por Imagem (CDPI)/DASA, Rio de J aneiro, RJ , Brazil.
}

However, theinvestigation of brain involvement in NMO by means of more advanced MRI techniques is still poorly developed. The concept of apparently normal white matter (ANWM) that is well established in MS, corresponding to the presence of normal signal intensity on white matter areas at conventional MRI sequences, however with significant inflammatory infiltration as histopathologically analyzed, is al so currently being defined in NMO. Thestudy with diffusion tensor imaging - a diffusion-based techniquethat allows analyzing the white matter microstructure integrity - has demonstrated to be useful in this process. A recent study published by the journal Radiology in April/2012 approached this subject ${ }^{(2)}$. By means of measurements of fractional anisotrophy (FA), radial diffusion (RD) and axial diffusion (AD), one can infer that diffuse microstructural changes occur in the apparently normal brain white matter tracts, going beyond the corticospinal tracts and optic radiations. Additionally, such study suggests that such changes are predominantly related to demyelination (RD-related FA change). In NMO, there has al ways been a tendency to consider that such loss of matter integrity was related to Wallerian degeneration secondary to distal lesions affecting the spinal cord and opticnerves. However, such study has demonstrated that the extent of the microscopic damage, otherwise "invisible" at conventional MRI techniques, is far greater and that, therefore, largeareas of theradiatecrown and particularly of the corpus call osum are affected.

In patients with NMO, the utilization of conventional and advanced MRI techniques in association with novel histopathological techniques has been useful to clarify the disease physiopathology, besides all owing a moreappropriateclassification of the disease within the spectrum of demyelinating disorders.

\section{REFERENCES}

1. Pires CE, Silva CM, Lopes FC, et al. Brain MRI abnormalities in Brazilian patients with neuromyelitis optica. J Clin Neurosci. 2012; 19:969- 74.

2. Rueda Lopes FC, Doring T, Martins C, et al. The role of demyelination in neuromyelitis optica damage: diffusion-tensor MR imaging study. Radiology. 2012;263:235-42. 